\title{
Does the lateral intercondylar ridge disappear in ACL deficient patients?
}

\author{
Carola F. van Eck $\cdot$ Kenneth R. Morse $\cdot$ Bryson P. Lesniak • Eric J. Kropf • \\ Michael J. Tranovich $\cdot$ C. Niek van Dijk $\cdot$ Freddie H. Fu
}

Received: 18 November 2009/Accepted: 22 December 2009/Published online: 20 January 2010

(C) The Author(s) 2010. This article is published with open access at Springerlink.com

\begin{abstract}
The aim of this study was to determine whether there is a difference in the presence of the lateral intercondylar ridge and the lateral bifurcate ridge between patients with sub-acute and chronic ACL injuries. We hypothesized that the ridges would be present less often with chronic ACL deficiency. Twenty-five patients with a chronic ACL injury were matched for age and gender to 25 patients with a sub-acute ACL injury. The lateral intercondylar ridge and lateral bifurcate ridge were scored as either present, absent, or indeterminate due to insufficient visualization by three blinded observers. The kappa for the three observers was .61 for the lateral intercondylar ridge and .58 for the lateral bifurcate ridge. The lateral intercondylar ridge was present in $88 \%$ of the sub-acute patients and $88 \%$ of the chronic patients. The lateral bifurcate ridge was present in $48 \%$ of the sub-acute and $48 \%$ of the chronic patients. This matched-pairs case-control study was unable to show a difference in the presence of the femoral bony ridges between patients with acute and chronic ACL injuries. The authors would suggest looking for the ridges as a landmark of the native ACL insertion site during ACL reconstruction in both acute and chronic ACL injuries.
\end{abstract}

C. F. van Eck · K. R. Morse · B. P. Lesniak

E. J. Kropf · M. J. Tranovich · F. H. Fu ( $\)$

Department of Orthopaedic Surgery, University of Pittsburgh

Medical Center, Kaufman Building Suite 1011,

3471 Fifth Avenue, Pittsburgh, PA 15213, USA

e-mail: ffu@upmc.edu

C. F. van Eck - C. N. van Dijk

Orthopaedic Research Centre Amsterdam,

Academic Medical Center, Meibergdreef 9,

1100 DD Amsterdam, The Netherlands
Keywords Lateral intercondylar ridge . Lateral bifurcate ridge .

Chronic anterior cruciate ligament injuries .

Anterior cruciate ligament reconstruction

\section{Introduction}

Recently, there has been an increase in interest in anatomic anterior cruciate ligament (ACL) reconstruction. This means using the native ACL insertion sites to determine the tunnel positions. It is hypothesized that this method will more closely restore the native anatomy of the ACL and may decrease the risk of developing early osteoarthritis. In acute patients, the remnants of the ACL can often be seen and used to determine the location of the insertion sites. However, in chronic patients, the remnants are often (partially) gone, and the surgeon has to rely on other landmarks, such as osseous landmarks, to determine the tunnel positions. The lateral intercondylar ridge is an osseous landmark that was first described by Clancy [6], who called it the residents' ridge. It is located on the medial wall of the lateral femoral condyle and runs from anterior to posterior with the knee in 90 degrees of flexion (operating position). Since its discovery, the ridge has been described in multiple anatomical studies. Purnell et al. [10] concluded that it clearly demarcates the anterior-most border of the origin of the ACL. Farrow et al. studied the morphology of the intercondylar notch in 200 human femurs and found the lateral intercondylar ridge to be present in 194 of them. Ferretti et al. [4] recently described an additional ridge perpendicular to the lateral intercondylar ridge, separating the anteromedial (AM) and posterolateral (PL) bundle insertion of the ACL; the lateral bifurcate ridge. 
It is suspected that the lateral intercondylar ridge exists because of osseous remodeling in response to stress from the ligament fibers, in accordance with Wolff's law [5]. This might be why the ridge is more clearly defined in young active people, as they put more strain on their ACL [8]. If this is true, when there is no ACL (in case of chronic insufficiency), the ridge could possibly gradually disappear. It has been suggested by surgeons that in patients with chronic ACL injury, the ridge is less visible or not visible at all. However, to our knowledge, this has never been proven in literature. Our hypothesis is that both the lateral intercondylar ridge and the lateral bifurcate ridge are present less often in patients with chronic ACL injury than in patients with acute ACL injury.

\section{Materials and methods}

This was a matched-pairs case-control study. Between 2005 and 2009, all patients who underwent ACL reconstruction after a chronic ACL injury were included. Chronic ACL injury was defined as the patient being ACL deficient for longer than 12 months before undergoing reconstructive surgery. These patients were then matched for gender and categorized age ( $<14$ years, 14-18, 18-23, $23-30,30-45$, or $45+$ ) to patients with sub-acute ACL injuries with \pm one age category accepted. Sub-acute was defined as a time shorter than 6 months between the ACL injury and the reconstructive surgery. A 6-month time period was left between the two groups in order to prevent overlap between the chronic and the sub-acute patients. Patients with previous injury to or surgery on the ipsilateral knee were excluded. Three orthopedic surgeons trained in sport medicine independently reviewed the arthroscopic footage of all patients and scored the presence of the lateral intercondylar ridge and lateral bifurcate ridge as either present, absent, or indeterminate due to insufficient visualization. Patient age, gender, body mass index (BMI), and the surgical delay were recorded. This study was approved by the Institutional Research Board (IRB).

\section{Statistical analysis}

The Kappa inter rater reliability for multiple raters was calculated. The ratings of one observer were used for further statistical analysis. When one of the ridges was scored as indeterminate, those data were considered missing. The demographic data were compared between the groups, using a paired t-test for continuous variables and the McNemar test for nominal variables. The presence of the lateral intercondylar and bifurcate ridge was expressed in percentages for both groups. The difference between the sub-acute and chronic group was calculated using the McNemar test. The a priori alpha level for statistical significance was set a $P<.05$.

\section{Results}

The kappa for the three observers was .61 for the lateral intercondylar ridge and .58 for the lateral bifurcate ridge.

Subject demographic data are displayed in Table 1. There was no difference between the groups for age, gender, and BMI. The surgical delay was different between the groups, with a mean of 2 months for the sub-acute subjects and 29 months for the chronic subjects $(P<.001)$.

The lateral intercondylar ridge was present in $88 \%$ of the sub-acute patients and $88 \%$ of the chronic patients (Fig. 1). It was scored as indeterminate in $4 \%$ and absent in $8 \%$ of the subjects in both groups. The lateral bifurcate ridge was present in $48 \%$ of both the sub-acute and chronic patients (Fig. 1). It was scored as indeterminate in 20 and $24 \%$ and absent in 32 and $28 \%$ of the sub-acute and chronic subjects, respectively (Figs. 2, 3).

Table 1 Demographic data of the included subjects

\begin{tabular}{|c|c|c|c|c|c|}
\hline & \multicolumn{2}{|l|}{ Acute } & \multicolumn{2}{|l|}{ Chronic } & \multirow[t]{2}{*}{$P$ value } \\
\hline & $\begin{array}{l}\text { Mean } \\
\text { or ratio }\end{array}$ & Range & $\begin{array}{l}\text { Mean } \\
\text { or ratio }\end{array}$ & Range & \\
\hline Age & 28 & $16-51$ & 27 & $16-63$ & NS \\
\hline \multicolumn{6}{|l|}{ Gender } \\
\hline Male: female & $10: 15$ & & $10: 15$ & & NS \\
\hline BMI & 25 & $20-38$ & 26 & $20-38$ & NS \\
\hline Surgical delay & 2 & $1-6$ & 29 & 12-109 & $<.001^{\neq}$ \\
\hline
\end{tabular}

$S D$ standard deviation, $B M I$ body mass index

$\neq$ The surgical delay was significantly different between the chronic and sub-acute group

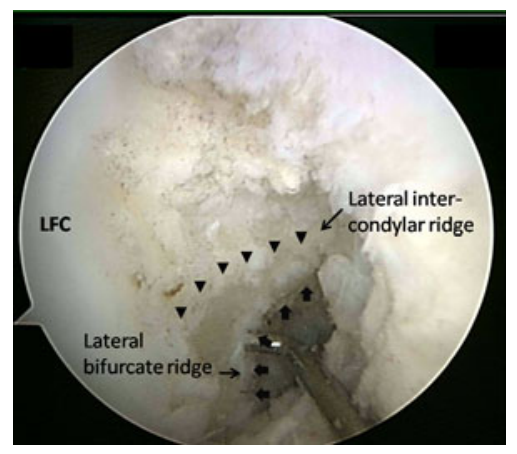

Fig. 1 Arthroscopic anteromedial portal view of the right knee in $90^{\circ}$ of flexion. Both the lateral intercondylar ridge and lateral bifurcate ridge are present 
For objects that were scored as indeterminate, the presence of the ridges could not be accurately determined. Therefore, for further statistical analysis, these patients were considered missing data, leaving 23 paired subjects in each group for comparison of the presence of the lateral intercondylar ridge. There was no difference between the sub-acute group and the chronic group $(P=1.00)$. Fifteen paired subjects in each group were available for comparison of the presence of the lateral bifurcate ridge. There was no difference between the sub-acute group and chronic group $(P=1.00)$

\section{Discussion}

The most important finding of the present study was that it was unable to identify a difference in the presence of the femoral bony ridges between patients with acute and chronic ACL injuries. It showed that the lateral intercondylar ridge is present in $88 \%$ of both acute and chronic ACL injuries. The lateral bifurcate ridge is present in $48 \%$

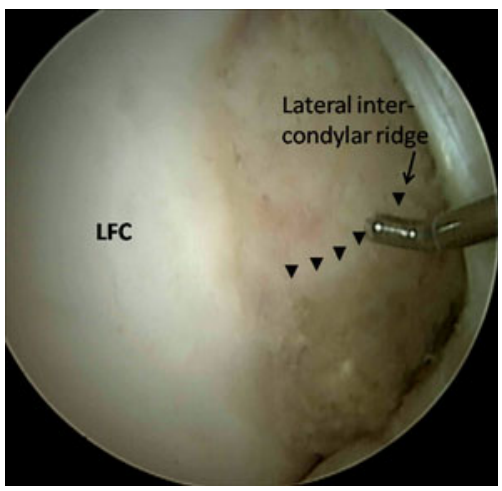

Fig. 2 Arthroscopic anteromedial portal view of the right knee in $90^{\circ}$ of flexion. The lateral intercondylar ridge is present, but the lateral bifurcate ridge is absent

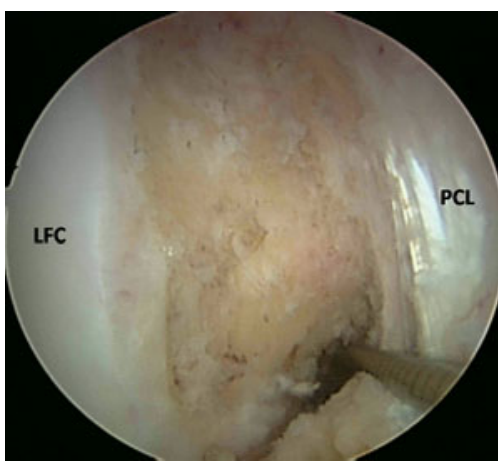

Fig. 3 Arthroscopic anteromedial portal view of the right knee in $90^{\circ}$ of flexion. Both the lateral intercondylar ridge and lateral bifurcate ridge are absent of acute and chronic patients. Farrow et al. [2] could identify the lateral intercondylar ridge in $97 \%$ of their specimens. However, these were femurs from a museum collection that had no soft tissue left and were studied in real time without the use of an arthroscope. It was not known if these subjects had ACL injuries.

A limitation of this study is that the presence of the ridges was determined from arthroscopic videos, not during the surgery by the observer. Therefore, the observer was unable to personally maneuver the scope and probe the ridges to determine their presence. However, we did find good agreement for the lateral intercondylar ridge among the three observers. The inter rater agreement for the lateral bifurcate ridge was only moderate. A possible explanation for this is that the observers found that this ridge was more difficult to visualize, resulting in the ridge often being scored as indeterminate. Based on the good inter rater agreement for the lateral intercondylar ridge, we felt it was justified to use the ratings of one observer for statistical analysis purposes. During this statistical analysis, an indeterminate score was considered as missing data. There were two patients in which the lateral intercondylar ridge could not be determined, one in the sub-acute group and one in the chronic group. Unfortunately, these two subjects were not paired to one another, thus when the paired analysis was done, two subjects in each group were excluded from the analysis, resulting in 23 patients in both groups, instead of 25. A similar situation occurred for the lateral bifurcate ridge, resulting in merely 15 available subjects in each group for that comparison.

Another limitation of the current study is the small sample size. This is attributed to the demographics of the practice sampled in this study, which primarily consists of acute ACL injuries. With the available sample size of 25 subjects per group, we were unable to find any difference in the presence of both ridges. In order to conclude that there is no difference in the presence of the ridges between patients with acute and chronic ACL injuries, a post-hoc power analysis was performed with a desired power of .90 . To find a $5 \%$ difference in the presence of the intercondylar ridge, assuming that the ridge is present in $90 \%$ of the patients with an acute ACL injury, we would need over 500 subjects per group. Based on our results and power analysis, we believe that the observed difference between acute and chronic patients is not clinically relevant.

A strong point of this study was that it was a matchedpairs case-control study. There was no influence of gender or age on the results of this study. An explanation for the inability of the current study to find a difference in the presence of the ridges between patients with chronic and acute ACL injuries could be that the presence of the ridges is influenced by other factors. One of which could be age. It is possible that more pronounced ridges may result as 


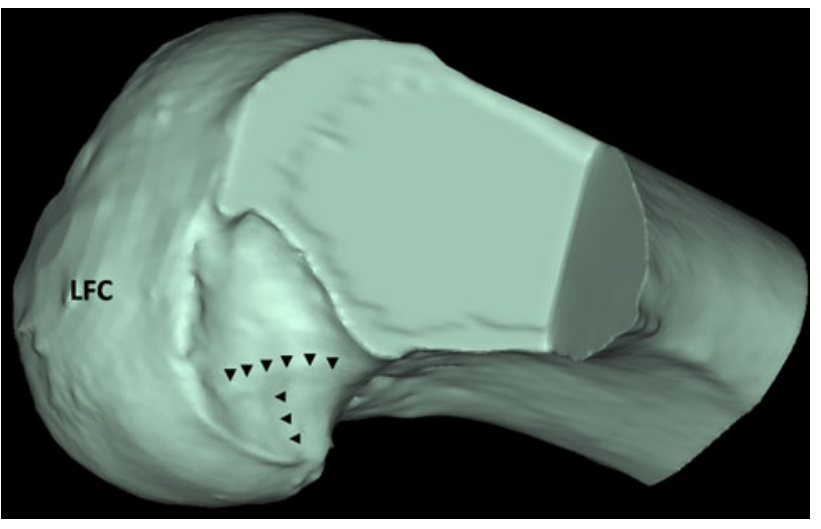

Fig. 4 Three-dimensionally reconstructed CT-scan of the right femur of a subject without ACL injury. Both the lateral intercondylar ridge and the lateral bifurcate ridge can be identified

people age, due to the sustained force of the ACL on the femur for a longer period of time. Another factor of influence could be an individual's activity level. This was not recorded in the present study. More active people have more force of the ACL, possibly resulting in greater ridge formation in young active people [8].

The range in surgical delay in the chronic group was 12 109 months, but it may take longer after ACL injury for the bony ridges to disappear. It is possible that this process takes many years. However, if this is the case, the clinical significance of when not to expect the ridge to be present would be small, since it is rare to perform reconstructive surgery on patients years after their injury. Furthermore, it is possible that there is no difference in the presence of the ridges between patients with acute and chronic ACL injuries. Surgeons who suggest that the ridge disappears may be biased by knowing the patient's history before starting the procedure. When the ACL remnants are no longer visible, it may be more difficult to locate the ridges. Sometimes, the ridge cannot be seen but only felt when the wall of the notch is carefully probed [5]. On the other hand, the observers in our study were specifically looking for both ridges and had no knowledge of patient's history.

If there is little difference in the presence of the ridges in acute and chronic ACL injuries, this would be beneficial to ACL reconstruction. The ridges are an important landmark for determining the native ACL insertion site [9]. In chronic injuries, when the femoral remnant has disappeared, the ridges can still be used to locate the ACL footprint. Even during ACL revision surgery, if the previous tunnel was drilled outside of the native insertion site, the ridges could inform the surgeon where to place the new tunnels [11]. The ridges can also be very useful during navigation-assisted reconstruction $[1,3,7,12]$. They can even be used to locate the native ACL insertion site in an uninjured knee, using three-dimensionally reconstructed computer tomography (CT) scans (Fig. 4).

\section{Conclusions}

The aim of this matched-pairs case-control study was to determine whether there is a difference in the presence of the lateral intercondylar ridge and the lateral bifurcate ridge between patients with acute and chronic ACL injuries. We hypothesized that the ridges would be present less often after chronic ACL deficiency. There was good agreement on the presence of the lateral intercondylar ridge and moderate agreement on the presence of the lateral bifurcate ridge. We found that the lateral intercondylar ridge was present in $88 \%$ of the acute patients and $88 \%$ of the chronic patients. The lateral bifurcate ridge was present in $48 \%$ of the acute patients and $48 \%$ of the chronic patients. To definitively conclude that there is no difference in the presence of the ridges between acute and chronic ACL injuries, more than 1000 subjects would be needed. We believe that such a small difference would probably not be clinically significant. The authors would suggest looking for the ridges as a landmark of the native ACL insertion site during ACL reconstruction in both acute and chronic ACL injuries.

Acknowledgments The authors did not receive any outside funding or grants directly related to the research presented in this manuscript. Our department receives funding from Smith and Nephew to support research related to reconstruction of the ACL. The research that forms the basis for this manuscript was done at our institution.

Open Access This article is distributed under the terms of the Creative Commons Attribution Noncommercial License which permits any noncommercial use, distribution, and reproduction in any medium, provided the original author(s) and source are credited.

\section{References}

1. Colombet PD, Robinson JR (2008) Computer-assisted, anatomic, double-bundle anterior cruciate ligament reconstruction. Arthroscopy 24:1152-1160

2. Farrow LD, Chen MR, Cooperman DR, Victoroff BN, Goodfellow DB (2007) Morphology of the femoral intercondylar notch. J Bone Joint Surg Am 89:2150-2155

3. Ferretti A, Monaco E, Labianca L, Conteduca F, De Carli A (2008) Double-bundle anterior cruciate ligament reconstruction: a computer-assisted orthopaedic surgery study. Am J Sports Med 36(4):760-766

4. Ferretti M, Ekdahl M, Shen W, Fu FH (2007) Osseous landmarks of the femoral attachment of the anterior cruciate ligament: an anatomic study. Arthroscopy 23:1218-1225

5. Fu FH, Jordan SS (2007) The lateral intercondylar ridge-a key to anatomic anterior cruciate ligament reconstruction. J Bone Joint Surg Am 89:2103-2104

6. Hutchinson MR, Ash SA (2003) Resident's ridge: assessing the cortical thickness of the lateral wall and roof of the intercondylar notch. Arthroscopy 19:931-935

7. Ishibashi Y, Tsuda E, Fukuda A, Tsukada H, Toh S (2006) Future of double-bundle anterior cruciate ligament (ACL) reconstruction: 
incorporation of ACL anatomic data into the navigation system. Orthopedics 29:S108-S112

8. Liu RW, Farrow LD, Messerschmitt PJ, Gilmore A, Goodfellow DB, Cooperman DR (2008) An anatomical study of the pediatric intercondylar notch. J Pediatr Orthop 28:177-183

9. Martins CAQ, Kropf EJ, Shen W, van Eck CF, Fu FH (2008) The concept of anatomic anterior cruciate ligament reconstruction. Oper Tech Sports Med 16:104-115

10. Purnell ML, Larson AI, Clancy W (2008) Anterior cruciate ligament insertions on the tibia and femur and their relationships to critical bony landmarks using high-resolution volume-rendering computed tomography. Am J Sports Med 36:2083-2090

11. Shen W, Forsythe B, Ingham SM, Honkamp NJ, Fu FH (2008) Application of the anatomic double-bundle reconstruction concept to revision and augmentation anterior cruciate ligament surgeries. J Bone Joint Surg Am 90 Suppl 4:20-34

12. Steckel H, Murtha PE, Costic RS, Moody JE, Jaramaz B, Fu FH (2007) Computer evaluation of kinematics of anterior cruciate ligament reconstructions. Clin Orthop Relat Res 463:37-42 